\title{
NOTA SOBRE LOS PRIMEROS REGISTROS HISTÓRICOS DE DOS PLANTAS INVASORAS EN LA PENÍNSULA DE BAJA CALIFORNIA: CRÓNICA DEL MISIONERO JESUITA MIGUEL DEL BARCO
}

\author{
Pedro P. Garcillán ${ }^{1,3}$ y Jon P. Rebman ${ }^{2}$ \\ ${ }^{1}$ Centro de Investigaciones Biológicas del Noroeste, Av. Instituto Politécnico \\ Nacional 195, Colonia Playa Palo de Santa Rita; 23096 La Paz, \\ Baja California Sur, México. \\ ${ }^{2}$ San Diego Natural History Museum, P.O. Box 121390, \\ San Diego, CA 92112, USA. \\ ${ }^{3}$ Autor para la correspondencia: ppgarcillan@cibnor.mx
}

\section{RESUMEN}

La fecha de introducción de las plantas invasoras en nuevas regiones es de gran relevancia ecológica, evolutiva y biogeográfica, pero suele ser difícil de determinar. Las fuentes históricas de la época de colonización europea pueden contener datos relevantes sobre la llegada de taxones no nativos a América. Durante el Periodo Misional Jesuita (16971768) se establecen los primeros asentamientos humanos permanentes y se introduce la agricultura y la ganadería en la Península de Baja California (PBC). Se revisaron textos escritos por los misioneros de esta orden relativos a la PBC y se encontraron datos precisos en la crónica de Miguel del Barco sobre la presencia y abundancia, en la primera mitad del siglo XVIII, de dos plantas actualmente invasoras en la región: Arundo donax y Ricinus communis.

Palabras clave: botánica histórica, especies exóticas, invasión biológica.

\section{ABSTRACT}

The date of introduction of invasive species in new areas is of high ecological, evolutionary and biogeographical relevance, although it can be difficult to determine. Historical sources on the European colonization period may contain relevant data about the arrival of some non-native taxa in America. During the Jesuit 
missionary period (1697-1768) in the Peninsula of Baja California (PBC), the first permanent human settlements were established and agriculture and livestock were introduced. Written sources by Jesuit missionaries who were present in the PBC were reviewed and precise data were found in the chronicle of Miguel del Barco regarding the presence and abundance of two currently invasive plants Arundo donax and Ricinus communis, which were already present in the PBC by the first half of eighteenth century.

Key words: biological invasion, exotic species, historical botany.

Las plantas no nativas (exóticas, introducidas) son aquellas presentes en una región más allá de su área nativa debido al transporte de forma directa o indirecta por las actividades humanas (Blackburn et al., 2011). El tiempo de residencia de un taxon en la nueva área es de gran significación ecológica y evolutiva (e.g., Le Roux et al., 2011; Hui et al., 2013). El proceso de invasión biológica se desarrolla en etapas secuenciales (introducción, establecimiento, expansión, invasión) (Richardson et al., 2000; Blackburn et al., 2011), y el tiempo de residencia influye en el paso de unas fases a otras (La Sorte y Pyšek, 2009). El mismo ayuda a trazar las rutas y secuencia temporal de la introducción y expansión de las especies en nuevas regiones y permite contextualizar la biogeografía de las invasiones biológicas (Le Roux et al., 2011). Desafortunadamente, la fecha de introducción de tales elementos suele ser difícil de determinar y con frecuencia solo se puede establecer un tiempo mínimo de residencia con base en las primeras colectas o en fuentes históricas confiables (Rejmánek et al., 2005). La expansión colonizadora europea a partir del siglo XV abre un periodo de intenso intercambio biológico a nivel mundial (Crosby, 2003). En México, la época colonial (1521-1810) ha sido señalada por la intensa llegada de especies nuevas (Challenger, 1998), aunque su fecha y lugar de introducción se desconocen en la mayoría de los casos (Sánchez-Blanco et al., 2012).

La península de Baja California (PBC) combina la existencia de barreras biogeográficas naturales y un proceso de colonización europea bien establecido espacial y temporalmente, sobre todo en su primer siglo. Tales características la hacen propicia para delimitar los tiempos de llegada de algunas especies no nativas en esta etapa inicial. Descubierta en 1533, la colonización de la península se logra exitosamente a partir de 1697, bajo control exclusivo de los misioneros jesuitas presentes en ella hasta su expulsión en 1768 (Martínez, 2005). Este periodo de actividad de la mencionada orden religiosa (1697-1768) es de importancia clave desde la perspectiva 
de las invasiones biológicas, ya que con ella se establecen por primera vez asentamientos humanos permanentes y se introducen la agricultura y la ganadería.

El propósito del presente estudio es identificar las plantas actualmente invasoras en la PBC que llegaron en la época de colonización jesuita. Se han señalado numerosas especies introducidas como cultivos en las misiones jesuitas, en su mayoría propias de la agricultura mediterránea, aunque también algunas nativas de México, y que han perdurado en gran medida en los sistemas agrícolas de los oasis bajacalifornianos (Nabhan et al., 2010; de Grenade y Nabhan, 2013a,b). Sin embargo, el objeto del presente trabajo se dirige a las plantas no nativas que de acuerdo con Garcillán et al. (2013) tienen actualmente carácter invasor (18 de 230 especies introducidas naturalizadas). El comportamiento invasivo fue determinado por estos autores con base en altas densidades de sus poblaciones observadas en campo, al menos localmente, y su elevada capacidad de dispersión y expansión (Garcillán et al., 2013).

Se revisaron reportes, cartas y relaciones de los misioneros jesuitas que estuvieron en la PBC y solo se encontró información sobre este tipo de elementos en la Historia natural y crónica de la Antigua California, manuscrito escrito por el religioso Miguel del Barco alrededor de 1780 en su exilio en Bolonia y publicado por primera vez en 1988 en la excelente edición de Miguel León-Portilla (Del Barco, ca. 1780). Miguel del Barco permaneció 30 años (1738-1768) en la misión de San Javier, situada a $20 \mathrm{~km}$ al oeste de Loreto, en el centro de Baja California Sur, y su manuscrito es sin duda la fuente de historia natural más detallada y fidedigna correspondiente al periodo jesuita. A continuación se presentan extractos del texto que constatan el establecimiento ya en el siglo XVIII de dos especies actualmente invasoras en la PBC: el carrizo (Arundo donax) y la higuerilla (Ricinus communis).

\section{Arundo donax L. (Poaceae):}

Asimismo en tales parajes [donde los arroyos conservan agua], si hay alguna tierra, nace mucho carrizo o caña, pero en toda la península es delgado y pequeño. $Y$ si en algunas partes hay algún carrizo grueso y largo, como el de España, es llevado de la costa de enfrente, el cual, transplantado en la California, crece también como en cualquier parte. El carrizo propio de la California es comúnmente no más grueso que los dedos de la mano (exceptuando el pólice, porque raro carrizo habrá tan grueso) y aún en algunas partes los hay más delgados, que dicho menor (del Barco, 1780 : 72).

El carrizo nativo (“delgado y pequeño" en palabras de del Barco) debe corresponder a Phragmites australis subsp. berlandieri, que es el único taxón de esta 
especie registrado en la península, y el "grueso y largo...llevado de la costa de enfrente" se identifica como Arundo donax. Se considera que A. donax es nativo de Asia y que posteriormente se extendió a la Cuenca del Mediterráneo, donde posiblemente se diversificó (Mariani et al., 2010). Desde esta última fue introducido en el Nuevo Mundo. Tarin et al. (2013) detectaron seis conjuntos clonales en Norteamérica, uno de ellos con amplia distribución geográfica que incluye México, Texas y California. Todos los individuos analizados de México (Durango, Zacatecas, Veracruz y Quintana Roo) pertenecieron al mismo grupo genético (Tarin et al., 2013). En 1820 el carrizo ya era abundante en el río Los Ángeles, California (Dudley, 2000). Según Greenlee (1992) fue llevado a California por los misioneros españoles, pero otros autores consideran que se introdujo a principio del siglo XIX para control de la erosión en canales de drenaje (Bell, 1997). El registro de del Barco sugiere que la relación entre las poblaciones de México y California encontradas por Tarin et al. (2013) puede tener como conexión aquellas introducidas en la península de Baja California.

Actualmente Arundo donax crece a lo largo de los ambientes riparios de la península y al menos en el noroeste de Baja California hemos observado que puede alcanzar altas densidades. En esta región posiblemente afecte la presencia de especies nativas (e.g., Platanus racemosa, Salix spp.) y las condiciones hidrológicas de estos ambientes, como ha sido reportado en otros estudios (Coffman, 2007; Giessow et al., 2011).

\section{Ricinus communis L. (Euphorbiaceae):}

Con demasiada frecuencia, se halla en algunos parajes de los arroyos, o sus cercanias, un vegetal que, ni puede contarse entre las yerbas, porque dura algunos años con vida, ni entre las matas lignosas que forman los matorrales, porque el tronco de ésta, que llaman higuerilla, nunca llega a ser perfectamente madera... Sus hojas tienen alguna semejanza con las de la higuera, aunque son muy redondas, y quizá por eso le dieron el nombre de higuerilla... La fecundidad de la higuerilla es admirable: a un mismo tiempo tiene unos racimos ya secos por muy maduros, otros cercanos a madurar, otros verdes, ya menos adelantados, otros en flor y otros que van saliendo de nuevo. De esta suerte fácilmente se difunde esta semilla arroyo abajo, llevándola las crecientes en las lluvias y dejándola donde no se pierda. De un arroyo a otro la llevan los caballos en sus colas, porque, con las espinas que tiene en la corteza, fácilmente se enreda entre las cerdas (del Barco, 1780 : 112-113). 
La higuerilla (R. communis) es un arbusto perenne originario del este de África, cultivado desde hace varios miles de años en Egipto y ampliamente naturalizado alrededor del mundo (Scarpa y Guerci, 1982). Foster et al. (2010), en su estudio de poblaciones naturalizadas de Florida, no encuentran evidencia de una dispersión significativa posterior al evento inicial de introducción. Sugieren que las plantas de una determinada región pueden proceder de múltiples eventos de introducción, probablemente asociados a las actividades humanas, ya que las cápsulas son pesadas y las semillas son expulsadas mecánicamente solo a algunos metros de la planta madre (Espirito-Santo, 2007). A este respecto es fascinante la información de del Barco sobre su capacidad reproductiva y su dispersión por lluvias torrenciales a lo largo de los cauces y por caballos entre cauces. La existencia de abundante ganado de pastoreo libre ("alzado" en palabras de del Barco) desde el comienzo de la colonización jesuita (Baegert, 1979; León-Portilla, 1988) hasta nuestros días (Martínez-Balboa, 1980; Inegi, 1997) posiblemente ayudó a su dispersión de forma rápida. Actualmente la planta está presente a lo largo de la península en zonas con disturbio antrópico o natural como bordes de caminos, asentamientos humanos y zonas riparias. En el noroeste mediterráneo de la PBC y en su extremo sur tropical de la Región del Cabo es una especie muy frecuente y localmente de elevada densidad.

Con base en los datos contenidos en el manuscrito de del Barco se puede afirmar que ambas especies eran ya frecuentes en la península a mediados del siglo XVIII. Sin embargo no se puede precisar su fecha de introducción. Es más probable que ésta ocurriera en el periodo de colonización exitosa (finales del siglo XVII o principios del siglo XVIII), pero no se puede descartar que se realizara durante la época de exploración e intentos fallidos de colonización que ocurrieron a partir de 1533.

Finalmente, cabe observar que otras especies introducidas como cultivos en las misiones jesuitas (del Barco, 1780; Baegert, 1979) están actualmente naturalizadas como la palma datilera (Phoenix dactylifera) o localmente escapadas, como en el caso del olivo (Olea europea), parra (Vitis vinifera), maíz (Zea mays), trigo (Triticum aestivum), sandía (Citrullus lanatus) y calabaza (Cucurbita pepo) (Rebman et al., en prensa).

\section{AGRADECIMIENTOS}

Deseamos agradecer la cuidadosa revisión realizada por dos revisores anónimos cuyas sugerencias mejoraron significativamente el manuscrito original. 


\section{LITERATURA CITADA}

Baegert, J. J. 1979. Observations in Lower California. University of California Press. Berkeley, USA. http://ark.cdlib.org/ark:/13030/ft5r29n9xv/. Consultado 27 abril 2015.

Bell, G. P. 1997. Ecology and management of Arundo donax, and approaches to riparian habitat restoration in Southern California. In: Brock, J. H., M. Wade, P. Pyšek y D. Green (eds.). Plant Invasion: Studies from North America and Europe. Backhuys Publishers. Leiden, The Netherlands. pp. 104-114.

Blackburn,T. M., P. Pyšek, S. Bacher, J. T. Carlton, R. P. Duncan, V. Jarosik, J. R. U. Wilson y D. M. Richardson. 2011. A proposed unified framework for biological invasions. Trends Ecol. Evol. 26(7): 333-339.

Challenger, A. 1998. Utilización y conservación de los ecosistemas terrestres de México. Pasado, presente y futuro. Comisión Nacional para el Conocimiento y Uso de la Biodiversidad, Instituto de Biología, Universidad Nacional Autónoma de México y Sierra Madre, S.C. México, D.F., México. 847 pp.

Coffman, G. C. 2007. Factors influencing invasion of giant reed (Arundo donax) in riparian ecosystems of Mediterranean-type climate regions. Ph.D. dissertation. University of California, Los Angeles, USA. 282 pp.

Crosby, A. 2003. The Columbian exchange biological and cultural consequences of 1492. 30th Anniversary Edition. Praeger, Westport, USA. 283 pp.

De Grenade, R. y G. P. Nabhan. 2013a. Baja California Peninsula oases: An agro-biodiversity of isolation and integration. Appl. Geogr. 41: 24-35.

De Grenade, R. y G. P. Nabhan. 2013b. Agrobiodiversity in an oasis archipelago. J. Ethnobiol. 33(2): 203-236.

del Barco, M. ca. 1780. Correcciones y adiciones a la historia o noticia de la California en su primera edición de Madrid, año de 1757. (Editado por M. León-Portilla como Historia natural y crónica de la Antigua California. Serie historiadores y cronistas de Indias/3, Instituto de Investigaciones Históricas, Universidad Nacional Autónoma de México, 1988, México D.F.). 482 pp.

Dudley, T. L. 2000. Arundo donax L. In: Bossard, C. C., J. M. Randall y M. C. Hoshovsky (eds.). Invasive plants of California's Wildlands. University of California Press. Berkeley, USA. pp. 53-58.

Espirito-Santo, M. M. 2007. Secondary seed dispersal of Ricinus communis Linnaeus (Euphorbiaceae) by ants in secondary growth vegetation in Minas Gerais. Revista Árvore 31: 1013-1018.

Foster, J. T., G. J. Allan, A. P. Chan, P. D. Rabinowicz, J. Ravel, P. J. Jackson y P. Keim. 2010. Single nucleotide polymorphisms for assessing genetic diversity in castor bean (Ricinus communis). BMC Plant Biol. 10: 1-13. http://www.biomedcentral.com/14712229/10/13. Consultado 24 febrero 2014.

Garcillán, P. P., J. L. León de la Luz, J. Rebman y J. Delgadillo. 2013. Plantas no nativas naturalizadas de la península de Baja California. Bot. Sci. 91(4): 461-475.

Giessow, J. H., J. Casanova, R. Leclerc, R. MacArthur, G. Fleming y J. Giessow. 2011. Arundo donax distribution and impact report March 2011. California Invasive Plant Council, USA. 238 pp. http://www.cal-ipc.org/ip/research/arundo/index.php Consultado 11 de febrero 2014. 
Greenlee, J. 1992. The Encyclopedia of ornamental grasses. Michael Friedman Publishing Group, Inc. New York, USA. 186 pp.

Hui, C., D. M. Richardson, P. Pyšek, J. J. Le Roux, T. Kucera y V. Jarošík. 2013. Increasing functional modularity with residence time in the co-distribution of native and introduced vascular plants. Nat. Commun. 4, artículo 2454: 1-8. DOI: 10.1038/ ncomms 3454

Inegi. 1997. El ganado bovino en el Estado de Baja California Sur. Instituto Nacional de Estadística y Geografía. Aguascalientes, México. 91 pp.

La Sorte, F. A. y P. Pyšek. 2009. Extra-regional residence time as a correlate of plant invasiveness: European archaeophytes in North America. Ecology 90: 2589-2597.

Le Roux, J. J., G. K. Brown, M. Byrne, J. Ndlovu, D. M. Richardson, G. D. Thompson y J. R. U. Wilson. 2011. Phylogeographic consequences of different introduction histories of invasive Australian Acacia species and Paraserianthes lophantha (Fabaceae) in South Africa. Divers. Distrib. 17: 861-871.

Mariani, C., R. Cabrini, A. Danin, P. Piffanelli, A. Fricano, S. Gomarasca, M. Dicandilo, F. Grassi y C. Soave. 2010. Origin, diffusion, and reproduction of the giant reed (Arundo donax L.): A promising weedy energy crop. Ann. Appl. Biol. 157: 191-202.

Martínez, P. L. 2005. Historia de Baja California. 4ª Edición. Universidad Autónoma de Baja California, Mexicali, México. 716 pp.

Martínez-Balboa, A. 1980. La ganadería en Baja California Sur. Volumen 1. Editorial J.B., La Paz, México. 229 pp.

Nabhan, G. P., J. García, R. Routson, K. Routson y M. M. Cariño-Olvera. 2010. Desert oases as genetic refugia of heritage crops: Persistence of forgotten fruits in the Mission orchards of Baja California, México. Int. J. Biodivers. Conserv. 2: 56-69.

Rebman, J.P., J. Gibson y K. Rich. En prensa. Annotated checklist of the vascular plants of Baja California, Mexico. Proc. San Diego Soc. of Nat. Hist.

Rejmánek, M., D. M. Richardson y P. Pyšek. 2005. Plant invasions and invasibility of plant communities. In: van der Maarel, E. (ed.). Vegetation ecology. Blackwell Science. Oxford, UK. pp. 332-355.

Richardson, D. M., P. Pyšek, M. Rejmánek, M. G. Barbour, F. D. Panetta y C. J. West. 2000. Naturalization and invasion of alien plants: concepts and definitions. Divers. Distrib. 6: 93-107.

Sánchez-Blanco, J., C. Sánchez-Blanco, M. Sousa S. y F. Espinosa-García. 2012. Assessing introduced Leguminosae in Mexico to identify potentially high-impact invasive species. Acta Bot. Mex. 100: 41-77.

Scarpa, A. y A. Guerci. 1982. Various uses of the castor oil plant (Ricinus communis L.). A review. J. Ethnopharmacol. 5: 117-137.

Tarin, D., A. E. Pepper, J. A. Goolsby, P. J. Moran, A. Contreras-Arquieta, A. E. Kirk y J. R. Manhart. 2013. Microsatellites uncover multiple introductions of clonal giant reed (Arundo donax). Invasive Plant Sci. Manag. 6(3): 328-338. 